\title{
Supporting information: Voltage-Induced Rearrangements in Atomic-Size Contacts
}

Markus Ring, ${ }^{\dagger}$ David Weber, ${ }^{\dagger}$ Patrick Haiber, ${ }^{\dagger}$ Fabian Pauly, ${ }^{\dagger, \dagger,}$ Peter Nielaba, ${ }^{\dagger}$ and Elke Scheer ${ }^{*} \dagger$

$\dagger$ Physics Department, University of Konstanz, 78457 Konstanz, Germany

$\ddagger$ Okinawa Institute of Science and Technology Graduate University, Onna-son, Okinawa 904-0495, Japan

INow at: Institute of Physics, University of Augsburg, 86135 Augsburg, Germany

E-mail: elke.scheer@uni-konstanz.de 


\section{Measurement Electronics}

To measure the electronic response of rearrangements in single-atom contacts, a simple yet versatile four-point measurement setup as shown in Figure S1 is used. A voltage bias is applied through a Yokogawa GS210 voltage source. The actual voltage at the sample is measured using a high-impedance differential voltage amplifier DLPVA from FEMTO. A FEMTO DLPCA transimpedance amplifier is used to measure the current. The motor used to drive the breaking mechanism is controlled by the Faulhaber motor controller MCDC 3002, and the superconducting magnet system is controlled by an Oxford Instruments IPS 120. Temperature monitoring and control is accomplished by a Lakeshore 335 .

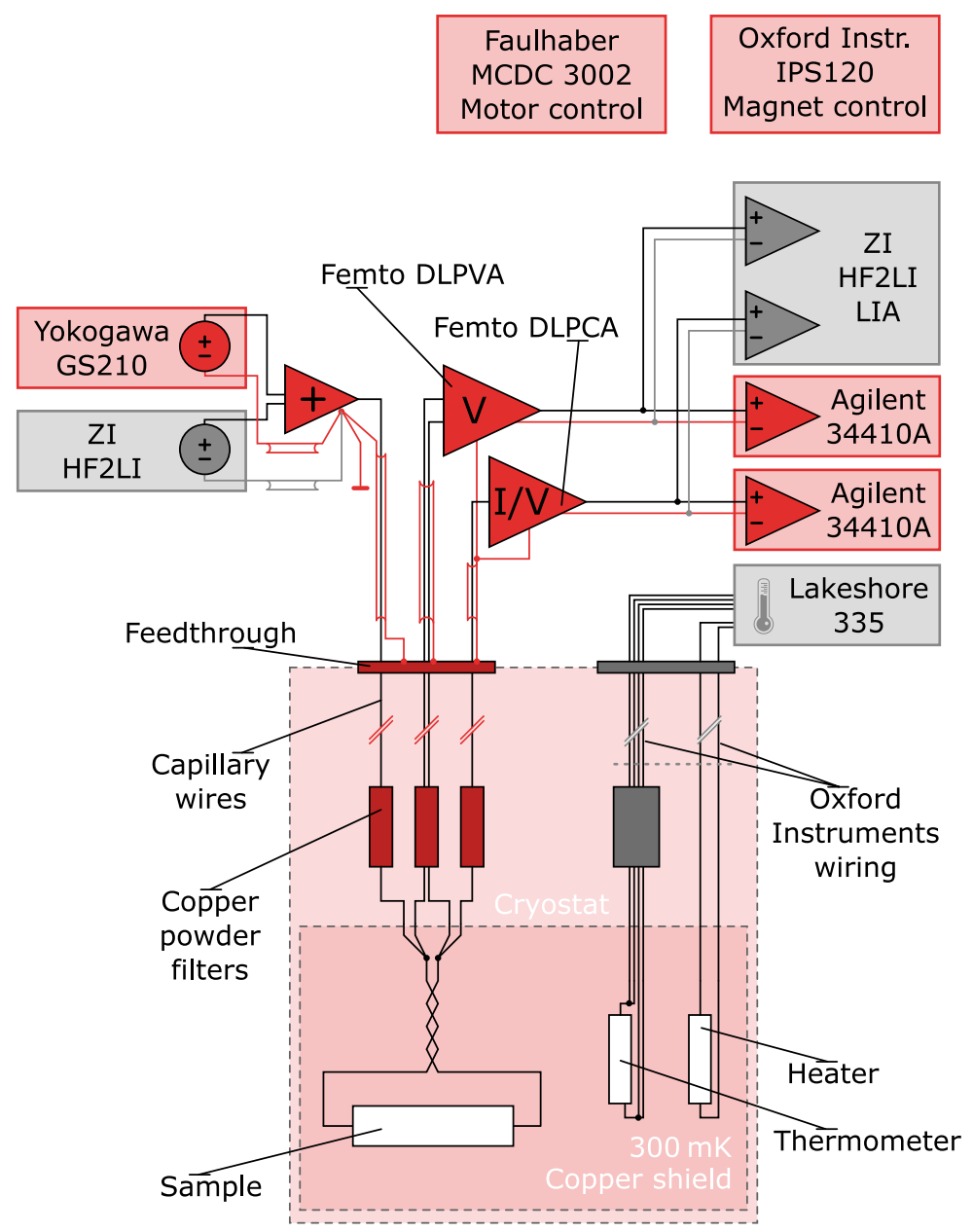

Figure 1: Schematics of the used four-point measurement electronics. Parts which are not in use are represented by grey color. 


\section{Conductance Histograms}

For sample characterization we first perform conductance measurements as a function of elongation of the bridges at low temperature $(T=4.2 \mathrm{~K})$ in cryogenic vacuum. From these data we calculate conductance histograms which are shown in Figure S2. They reveal the typical structure and preferred conductance values known from earlier measurements, ${ }^{1-4}$ obtained partially with different sample types. We then perform electromigration experiments on atomic-size contacts which have been adjusted mechanically also at low temperature. The typical switching currents are in the order of $200 \mu \mathrm{A}$ and the corresponding voltages amount to 5 to a $500 \mathrm{mV}$. The quality of the metallic contacts can be verified by conductance histograms. To this end, the contact is repeatedly opened to form a tunnel contact and deeply closed to a conductance of more than $50 \mathrm{G}_{0}$ to gather statistical information on the preferred conductance values. Binning the data according to their conductance yields information of how likely a conductance appears. Usually, the last peak before breaking the contact into the tunnel regime represents a single-atom contact with a conductance and a shape (length, slope) which is characteristic for a metal. ${ }^{1}$ Figure S2 presents conductance histograms of the four metals studied which are acquired at low temperature in cryogenic vacuum before starting the electromigration procedure. The plots are created from thousands of data points in hundreds of breaking curves. The recorded data fits well to measurements performed before on the respective metals. ${ }^{5-10}$ Through this comparison, we can assure a clean and pristine contact material.

\section{Examples of Voltage-Driven Rearrangements of All Met-} als

Figure 3 shows examples of electromigration experiments for all four metals investigated. Figure 3a shows the same data as in the main text (Figure 1c). The color code describes the temporal development from dark (beginning) to light (end) colors. The example in Figure 3c for $\mathrm{Al}$ contains a section of bivalued switching for about four cycles. Such data is excluded from the statistical analysis.

\section{Analysis of Experimental Data}

The raw voltage and current data is subject of automized analysis to ensure comparability of the results. Due to noise, two main criteria have to be fulfilled to accept an event:

- a sufficiently large $\Delta G=0.1 \mathrm{G}_{0}$ for $\mathrm{Pb}, \mathrm{Al}$, and $\mathrm{Al}(\mathrm{Cu})$ and 

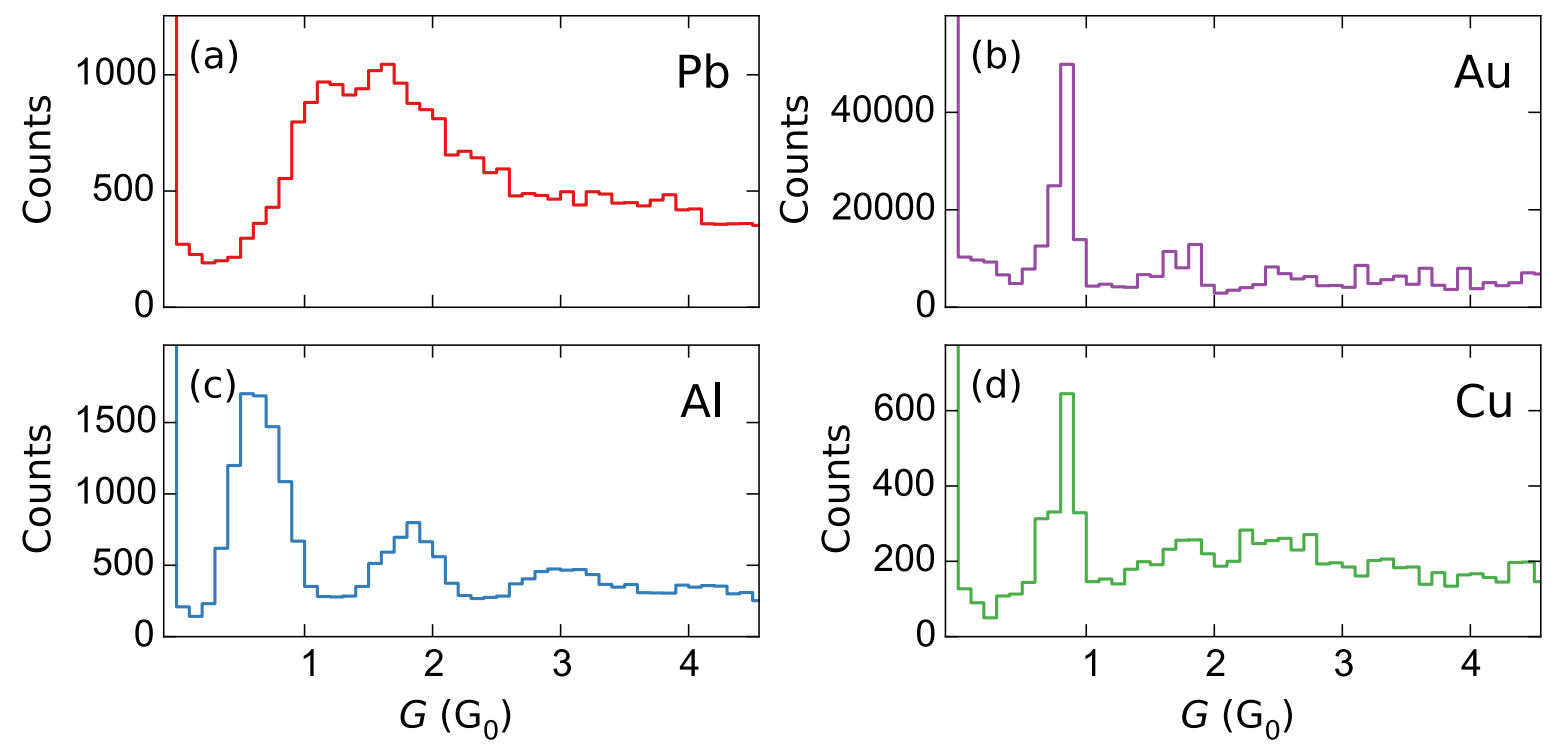

Figure 2: Conductance histograms of the four metals exhibiting the known preferred conductance steps in the few-atom regime. $\mathrm{Pb}$ and $\mathrm{Al}$ show their last stable configurations above and below the conductance quantum $G=1 \mathrm{G}_{0}$, respectively. The monovalent metals $\mathrm{Cu}$ and $\mathrm{Au}$ exhibit a maximum close to $G=1 \mathrm{G}_{0}$, interpreted as a signature of a single well transmitting channel. A tail of the first maximum to lower conductance values was observed before in lithographically fabricated samples and is attributed to disorder due to the sample fabrication procedure using thin films and reactive ion etching.

- more than $120 \mathrm{mV}(60 \mathrm{mV})$ applied to the $\mathrm{Au}$ and $\mathrm{Cu}$ (Al and $\mathrm{Pb})$, contact since the conductance determination $G=\frac{I}{V}$ becomes imprecise at low bias.

Additionally, events too close in time, and events triggered by the breaking of the contact are treated as artifacts and are removed.

Subsequently, the obtained threshold parameters were binned in the range of 0 to $180 \mu \mathrm{A}$. Using a Gaussian fit, the center and the width of the normalized distribution was determined according to Eq. S1.

$$
G(I, \alpha, \mu, \sigma)=\alpha \cdot e^{\frac{(I-\mu)^{2}}{2 \sigma^{2}}}
$$

\section{Simulation Setup}

For comparison with the experimental results we simulate stretching experiments with the help of molecular dynamics simulations and their conductance using density functional theory and nonequilibrium Green's functions. ${ }^{11,12}$ The initial configuration were slabs of metals in a perfect lattice in 100, 110 and 111 direction. These slabs were pulled in those directions 

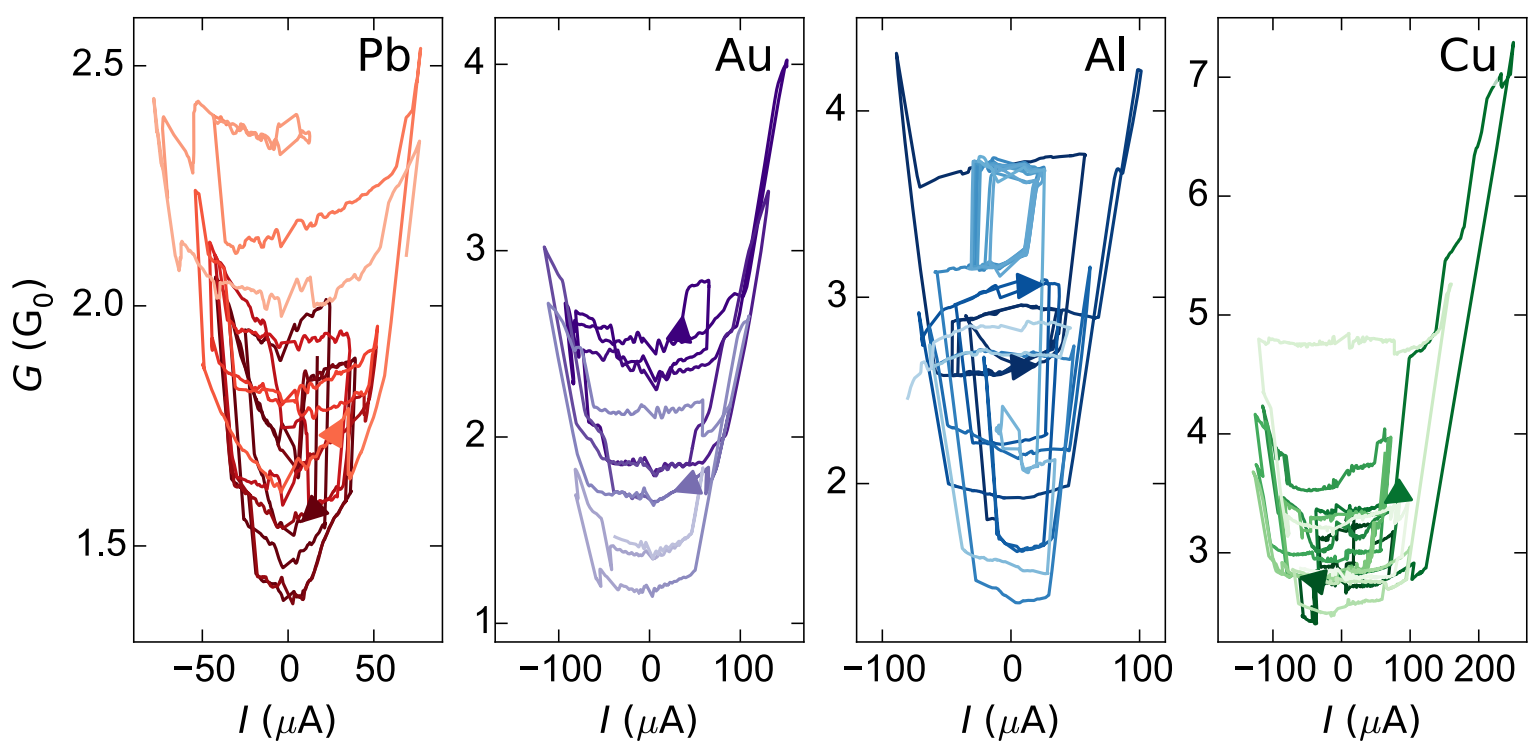

Figure 3: Typical electromigration measurements for the different metals. Plot of $G$ against $I$ revealing the temporal evolution of a contact in a compact way. The arrows indicate the direction of evolution while the colors are derived from the individual rearrangement cycles. Noisy data with $|V|<25 \mathrm{mV}$ is removed for clarity.

through molecular dynamics. These contacts were than relaxed again in DFT to get sample contacts for the simulation, see Figure 4 for some examples of relaxed contacts.

Examples of stretching curves, i.e. conductance as a function of distance, and conductance histograms compiled from this data are shown in Figure S6. Typical features known from the experiments, like the pronounced long last plateau and histogram maximum for Au at $1 \mathrm{G}_{0}$, the increasing conductance value on the plateaus for $\mathrm{Al}$, or the broad single-atom contact maximum for $\mathrm{Pb}^{1}$ are well reproduced.

To test the validity of the model applied to determine the mechanical properties, we also calculate the equilibrium phonon modes and phonon density of states and show them in Figure S7. Also here the agreement with literature and experiments is very good, in particular for $\mathrm{Al}$, where we will find discrepancies regarding the switching properties. Next, we calculate the runaway voltage, at which at least one phonon mode of the simulated contact is pumped.

\section{Switching Model}

As an important parameter, the runaway voltage $V_{r}$ is identified by a change of sign in the damping of individual modes - at voltages higher than this threshold, modes which were previously dampened are instead pumped by the scattering of the nonequilibrium electrons. 


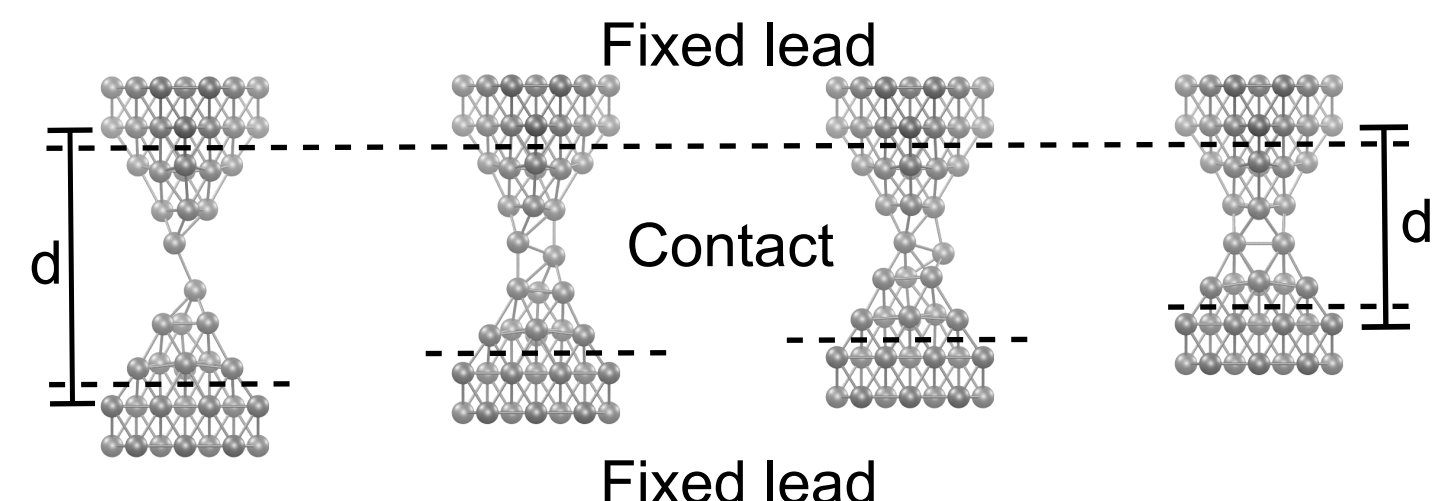

Figure 4: Example contact configurations of one $\mathrm{Pb}$ distance sampling. The crystal direction is 111 and the distances between the fixed leads are $d=19.7,17.8,16.5$ and $14.5 \AA$ from left to right.

The central investigation of this work is to elucidate, whether that runaway voltage is a relevant parameter for real systems, i.e. whether this calculated runaway voltage is comparable to experimental switching voltages and behaves similarly between metals and data sets. Similarity here indicates that the runaway voltage and therefore active pumping of phonon modes is a relevant mechanism for nanoscale switching and electromigration.

To illustrate the properties of the Langevin equation relevant to switching - namely anisotropy of the trajectories and effective heat bath - we present a simple 2D model system consisting of a particle in the $x y$-plane, the movement of which obeys a Langevin equation ${ }^{13,14}$ of the form:

$$
m \ddot{x}=-k x-\gamma \dot{x}+\sqrt{2 k_{b} T \gamma} W(t)
$$

The system propagates in a potential consisting of two harmonic minima at $\vec{x}_{1}=(1,1)$ and $\vec{x}_{-1}=(-1,-1)$. The relevant potential is switched at the line of same distance from both minima.

For this model simulation we neglect the generalized part of the Langevin equation and do not resolve time-scales below delta-correlated force-noise. Therefore, the force noise term $\sqrt{2 k_{B} T \gamma} W(t)$ fulfills the classical fluctuation-dissipation theorem. The friction couples the system to a heat bath with energy $k_{B} T$ simulated through a normally distributed Wigner process $W(t)$. Additionally the system is coupled through the friction and force noise terms to a nonequilibrium electronic system with energy scale $\propto V$.

The central ingredient of the model simulation are nonconservative forces. That means the dynamical matrix $k$ and the friction matrix $\gamma$ contain voltage-dependent anti-symmetric terms, which can turn the system nonconservative, allowing energy to be pumped into there in excess ability of the system to dissipate it. To simulate this the friction term in $\mathrm{x}$-direction 

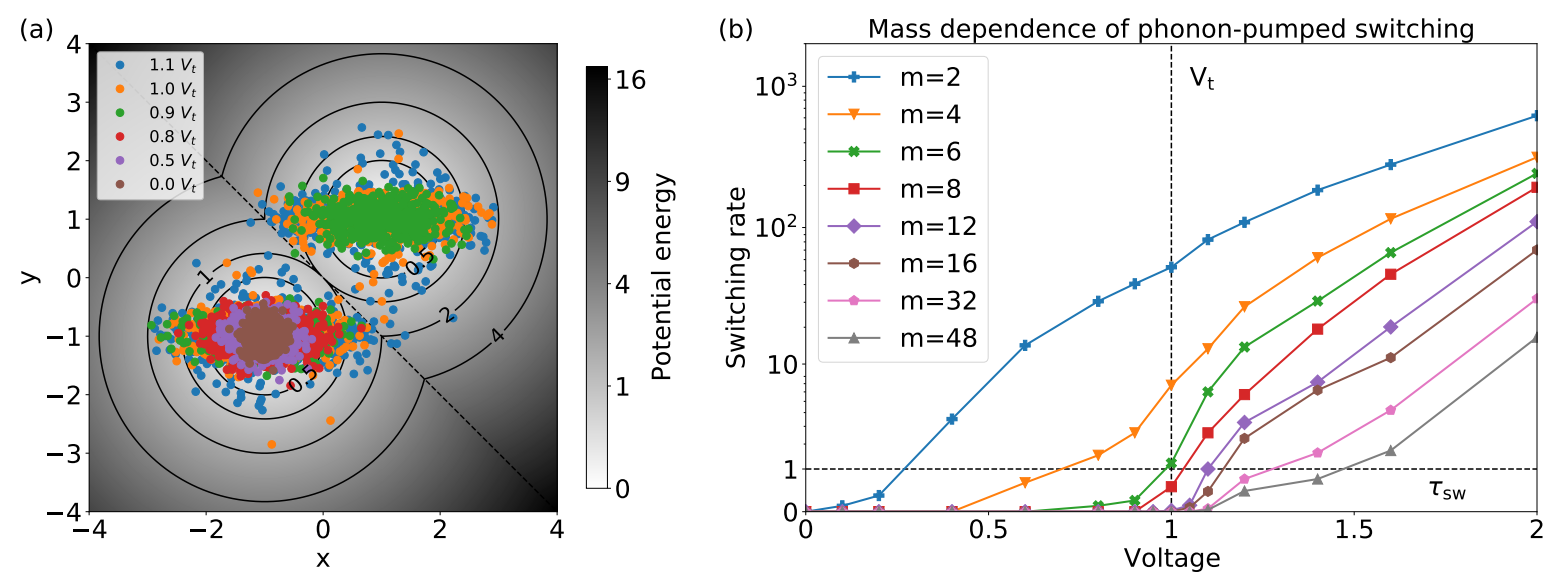

Figure 5: Sample trajectories and switching rates for model simulations.

is set to be voltage dependent in a form $\gamma(V)=\gamma_{0}-c \cdot V$ with $V_{t h r}=\frac{\gamma_{0}}{c}$ as the defined voltage at which the sign of the friction term changes from damping to pumping. The fluctuating term increases with voltage according to $\sqrt{2 k_{b} T \gamma_{0}+c_{D} \cdot c \cdot e V}$. Here $c$ is the strength of the voltage-friction coupling and $c_{D}$ encompasses the effect the voltage has on the random force. While $c$ has been calculated in the actual simulations, the effect on the fluctuating forces is considerably more involved and it is doubtful that a simple additive white noise term can encode the full behavior. ${ }^{14}$ For this model system we set $c=\gamma_{0}^{-1}$ to have a runaway voltage of 1 and $c_{D}=0.1$ in order to have a about equal contribution of thermal and electronic friction noise at the threshold in order to focus on the effect of the change of friction instead of the random force terms.

In Figure S5 a) simulated trajectories of this system are shown. With increasing voltage those trajectories grow more extended especially in the $(\mathrm{x})$ direction of decreasing friction. When the trajectory crosses the diagonal there is a high likelihood for relaxation into the other minimum.

Dividing eq S2 by the mass leaves only the acceleration on the left side. This determines the time-scale of the system, which then scales with the inverse of the mass. This changes the phonon frequencies $\sqrt{k / m}$ and momentum relaxation times $m / \gamma$, but leaves the overall extent of the trajectories independent of mass. The timescales are nonetheless changed by a factor $\propto m$, heavier particles move slower through those trajectories. This is a property of the overdamped classical system - in the ballistic limit the extent of the trajectory in a harmonic oscillator is proportional to the inverse square-root of the mass $\sigma_{r} \propto m^{-1 / 2}$, same as for the quantum-mechanical harmonic oscillator.

If one assumes a $m^{-1 / 2}$ dependence of the fluctuating forces, one gets a mass dependence of the switching rates which is not just a rescaling with time. This is shown in Figure S5 
b), where we assume a switching rate of 1 per one million time steps would be measured as a switching event in the experiment. There are two different exponential growth regimes before crossing the runaway voltage, enhancing the voltage acts as an effective temperature, making switching more likely. After crossing the runaway voltage, the mode leading to switching is effectively pumped, boosting the switching rate considerably. This could explain why the runaway voltage is a better predictor for the switching voltage of the heavier metals: The heavier metal needs the active pumping to switch in the observed timescales, while for the lighter metals thermal excitation can be sufficient.

\section{Runaway Voltage}

The switching voltage measured in experiment is a different physical property than the runaway voltage calculated in the simulations. There is a time scale gap of at least six orders of magnitude between the movement of electrons and individual atomic rearrangements (on the scale of picoseconds) and the long-term excitation leading to switching, which are assumed to be on the time scale of microseconds. This time-scale gap together with the comparatively expensive electronic structure calculation at each time step precludes a fully dynamical simulation of the switching process. In order to bridge that gap to obtain the simulated data, the electronic degrees of freedom are integrated into a Langevin equation for the mobile atoms:

$$
m \underline{\ddot{x}}=\underline{\underline{D}} \cdot \underline{\mathrm{x}}-\underline{\underline{R}} \cdot \underline{\dot{\mathrm{x}}}+\underline{\mathrm{F}}_{R}
$$

where $\underline{x}$ is the vector of the atomic coordinates, with its time derivatives acceleration and velocity. $\underline{\underline{D}}$ is the matrix of the force constants for small deviations from the equilibrium positions of the atoms, giving rise to vibrations and phonons, and $\underline{\underline{R}}$ is the friction matrix due to the electron-vibration coupling or electronic friction. In equilibrium they are related with an Einstein relation with the random force $\underline{\mathrm{F}}_{R}$. These matrices are calculated at zero bias with DFT, resulting in phonon frequencies and their damping.

The NEGF methods allows one to calculate the changes to these matrices by applying a voltage to the system, which is done up to first order in the voltage around the Fermi energy in the wide-band limit. This can be understood as the forces acting on the atoms caused by the inelastic scattering of the nonequilibrium electrons crossing the contact. At small voltages these energies are easily dissipated into the bulk, keeping the contact configurations stable. Only when, through an increasing voltage, the number of crossing electrons and scattering events increases beyond the capability of the contact to dissipate the excess energy a structure change is possible.

The runaway voltage is such a crossover point. It is determined by solving the eigenvalue 
problem posed by eq. 3 for increasing voltages. The runaway voltage is reached when the damping of one of the modes changes sign. If this happens this mode becomes unstable and the contact may switch into another configuration.

In the theory and simulation only the absolute value of the voltage $V$ is relevant. This is due to the NEGF construction using energy conservation for the scattering and not momentum conservation. In reality the direction of the voltage can be important for the specific contact. Although we assume that field effects are lost to the metallic nature of the contact, this may not be the case in these surface situations. To include the direction of the voltage and current in the theory, it would be necessary to extend the approach from purely energy conserving scattering to momentum conserving. This would then lead to the question of quasi-momentum conservation and scattering since the vibration of the atoms is in both directions, and most of the momentum transferred to the atoms is lost to the bulk. A calculation of the phonon transport into the bulk was used to calculate an excess kinetic energy at a voltage-direction-dependent position ${ }^{15}$ but this allowed no comparison with experiments, since kinetic energy cannot be resolved at atomic resolution.

\section{Joule Heating}

Structure changes on atomic to mesoscopic length scales are often ascribed to Joule heating - an increase in temperature of the structure due to dissipated energy by the current flowing through it. The temperature rise leads to an activated barrier crossing or and reduction of barriers between different possible positions of the atoms. On the atomic scale, several of the properties of this thermodynamic picture are no longer valid: The variance of energy is far from the thermodynamic limit, the periodicity and isotropy are broken by the surface, and the external bias and the random forces are colored instead of having a white power spectrum.

These properties lead to a behavior different from the usual kinetic picture of activated barrier crossing or barrier reduction, which is based on a thermal view. The 2D Langevin model presents an approach, where the trajectory explicitly does not cross at the minimal barrier. Instead the crossing is along the excitation direction. In that dynamical case with nonthermal, anisotropic movement, a barrier is not easily identifiable. The anisotropy of the trajectories in the model also comes from the friction and not from the underlying potential, although in a real contact there would of course be interaction between the specifics of the atomic structure and the friction.

The importance of those different properties for the behavior of real contacts can only be determined by comparing directly with experimental data. This is done in this work by 
comparing experimental switching voltages with simulated runaway voltages. The runaway voltages denote vibration modes turning unstable through one of the effects contributing to Joule heating, namely the change in the dynamical matrix and the friction kernel felt by the atoms due to the scattering of nonequilibrium electrons.

\section{Simulated Conductance Histograms}

Figure S6 presents simulated pull curves and conductance histograms for the four studied metals. All of those exhibit metal-specific features and behave in ranges similar to the experimental values. The simulated data set stays far below the numbers attainable in experiment, mostly due to the prohibitive cost of relaxing those structures.

$\mathrm{Pb}$ exhibits a broad peak between around $3 \mathrm{G}_{0}$ - which is quite different than the behavior in experiment, where there is a wide peak in between 1 and $2 \mathrm{G}_{0}$. This is mostly due to an extended plateau around $2-3 \mathrm{G}_{0}$ that is stable in all simulated pull and push curves. $\mathrm{Au}$ shows well developed conductance plateaus and a strong preference for contacts with integer multiples of the conductance quantum in the histogram - as is known from other simulations. ${ }^{16,17} \mathrm{Al}$ exhibits peaks below $1 \mathrm{G}_{0}$, around $2 \mathrm{G}_{0}$ and again at $4 \mathrm{G}_{0}$. The peak in Al below $1 \mathrm{G}_{0}$ is similar to experiment, same as the one around $2 \mathrm{G}_{0}$. The one around $4 \mathrm{G}_{0}$ is not due to one curve alone and is likely due to a preferred configuration in simulation. This is supported by the comparatively higher runaway voltage calculated for those contacts. $\mathrm{Cu}$ shows the same broad distribution as found in experiment for conductance values larger than $1 \mathrm{G}_{0}$. The $1 \mathrm{G}_{0}$ peak of the experiment could not be reproduced, since simulated contacts around the distance where those conductance values would be expected do not relax. If that is due to an comparatively flat free energy surface with many possible states as would be expected of the experimental peak or due to errors in the parametrization in simulation is a question for further investigation.

\section{Simulated Vibration and Damping Density of States}

Figure S7 presents the density of states (DOS) of the vibration frequency and the damping. The vibration energies fit to experimental phonon measurements ${ }^{18}$ and bulk simulation data, although the specific structure of the bulk material is not visible in the simulated surface structures. The damping of the vibration modes, which is connected with the electronvibration coupling, has not been measured directly. For all four metals there is a prominent peak and a long tail. The damping peak is around $40 \mu \mathrm{eV}$ for $\mathrm{Pb}, 80 \mu \mathrm{eV}$ for $\mathrm{Au}$ and $\mathrm{Al}$ and $150 \mu \mathrm{eV}$ for $\mathrm{Cu}$. Regarding the classification in noble $(\mathrm{Au}, \mathrm{Cu})$ and other metals $(\mathrm{Pb}, \mathrm{Al})$, the distribution in damping is broader for the noble metals and for the lighter materials. 

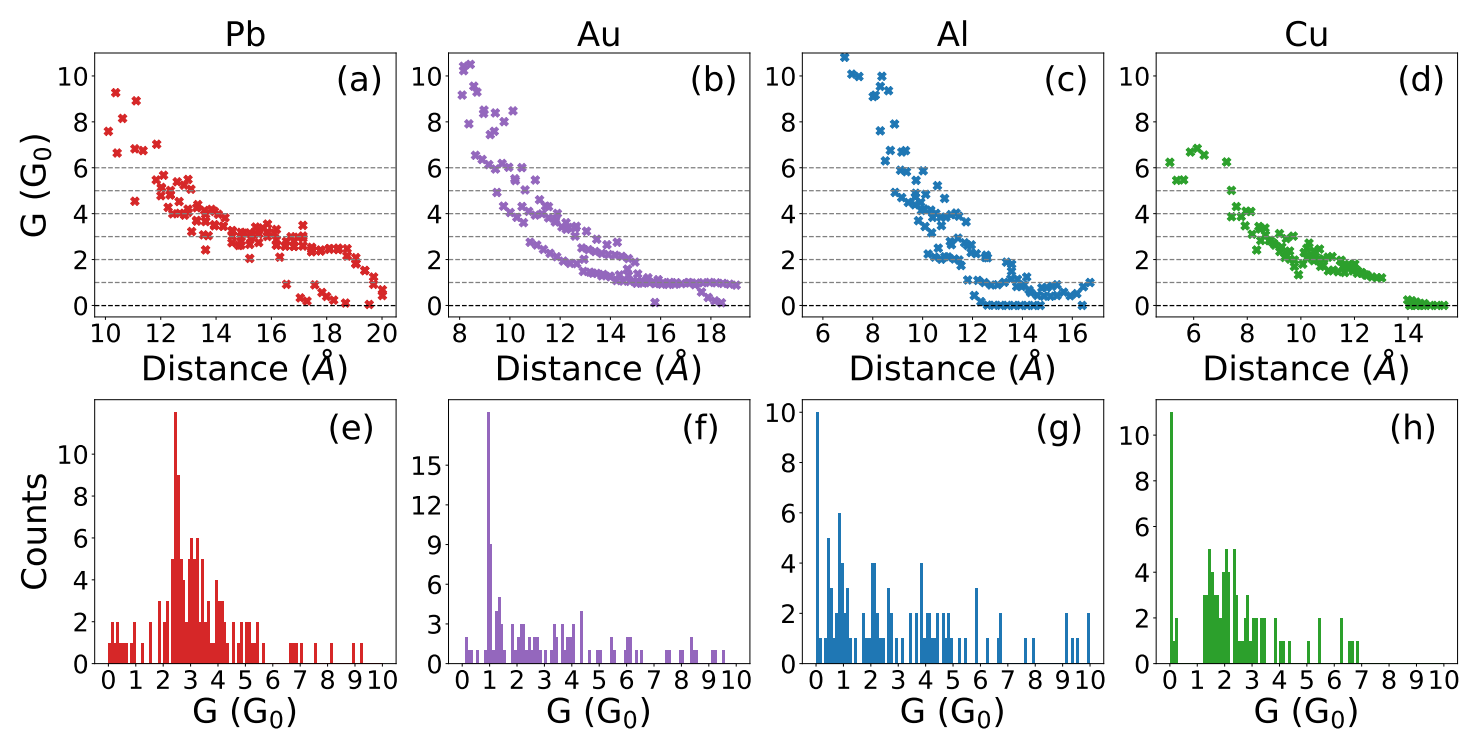

Figure 6: Aggregated pull curves and conductance histograms of the four simulated metals exhibiting conductance steps in the few-atom regime.

Overall this gives an ordering in damping variance of $\mathrm{Pb}<\mathrm{Al}<\mathrm{Au}<\mathrm{Cu}$. This behavior stronger and more varied damping is also visible in the high-damping tail of the materials, which has larger extent in the order given above.

\section{Computational Costs}

Although only a comparatively small number of atoms per contact are simulated, the calculations are quite expensive. This is primarily due to the high requirements for the structural relaxation necessary to calculate the vibration spectrum and the electron-vibration coupling with small errors within the simulation framework. For a typical contact it takes around a week of real time on a supercomputer node with 16 cores to relax the structure, accounting for more than $90 \%$ of the calculation time. The parallelization efficiency of a single contact of usual size drops below $80 \%$ with more than 16 cores. All together a single contact takes at the low end around $1000 \mathrm{~h}$ of CPU time to simulate. This has to be multiplied with the number of contacts needed to get a reasonable sample size considering the variability of the contacts and the process investigated. Overall the simulations presented in the paper took more than one million core hours to produce. 

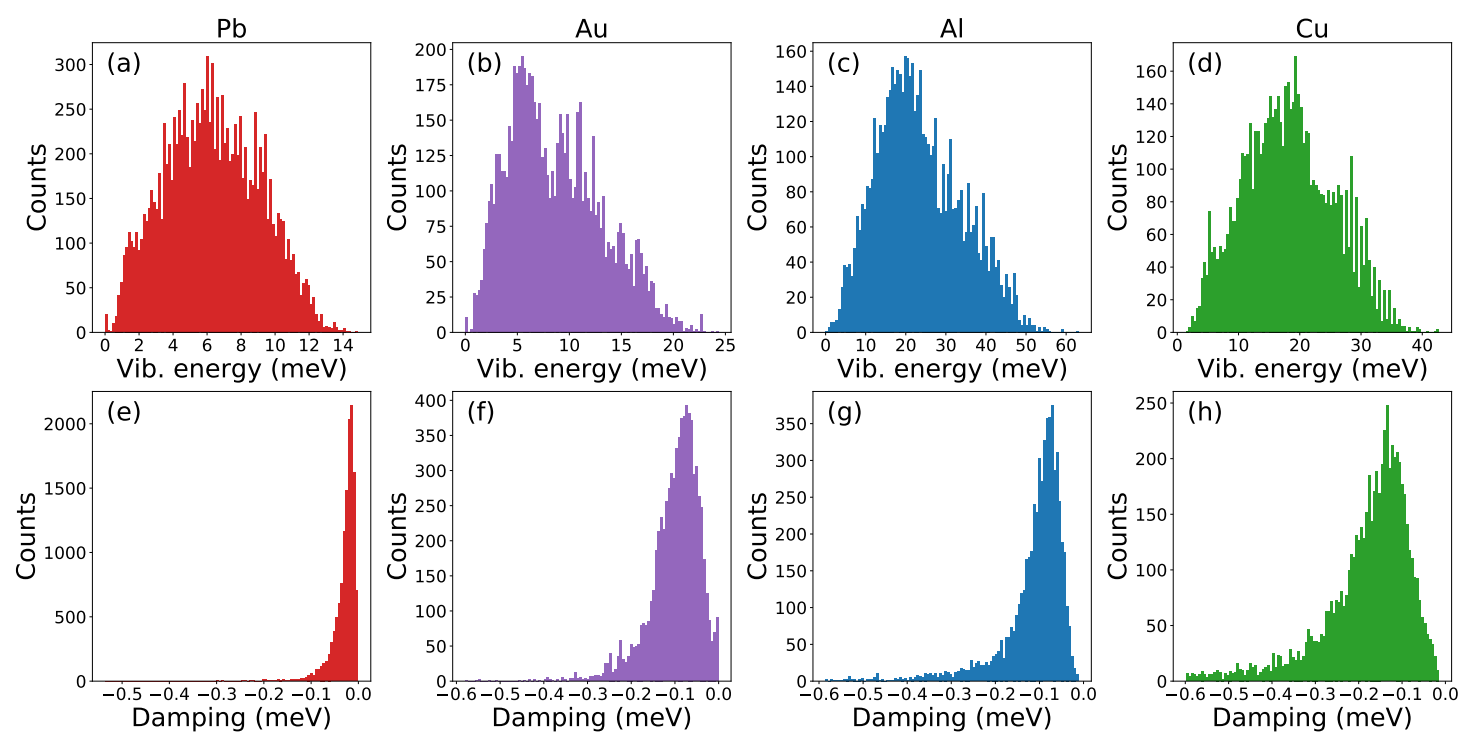

Figure 7: The density of states of the vibrations (top) and the damping (bottom) of the four simulated metals.

\section{References}

1. Agraït, N.; Levy Yeyati, A.; van Ruitenbeek, J. M. Quantum Properties of Atomic-Sized Conductors. Phys. Rep. 2003, 377, 81-279.

2. M. Krans, J.; J. Muller, C.; K. Yanson, I.; C. M. Govaert, T.; Hesper, R.; van Ruitenbeek, J. One-Atom Point Contacts. Phys. Rev. B 1993, 48, 14721-14724.

3. Scheer, E.; Agraït, N.; Cuevas, J. C.; Levy Yeyati, A.; Ludoph, B.; Martín-Rodero, A.; Bollinger, G. R.; van Ruitenbeek, J. M.; Urbina, C. The Signature of Chemical Valence in the Electrical Conduction through a Single-Atom Contact. Nature 1998, 394, 154-157.

4. Weber, D.; Scheer, E. Superconducting Properties of Lithographic Lead Break Junctions. Nanotechnology 2018, 29, 045703.

5. Yasuda, H.; Sakai, A. Conductance of Atomic-Scale Gold Contacts under High-Bias Voltages. Phys. Rev. B 1997, 56, 1069-1072.

6. Yanson, I. K.; Shklyarevskii, O. I.; Csonka, S.; van Kempen, H.; Speller, S.; Yanson, A. I.; van Ruitenbeek, J. M. Atomic-Size Oscillations in Conductance Histograms for Gold Nanowires and the Influence of Work Hardening. Phys. Rev. Lett. 2005, 95, 256806.

7. Yanson, A. I. Atomic Chains and Electronic Shells: Quantum Mechanisms for the Formation of Nanowires; Leiden University: Leiden, 2001. 
8. Makk, P.; Csonka, S.; Halbritter, A. Effect of Hydrogen Molecules on the Electronic Transport through Atomic-Sized Metallic Junctions in the Superconducting State. Phys. Rev. B 2008, 78, 045414 .

9. Schirm, C. Einfluss Hoher Ströme Auf Atomare Kontakte. Ph.D. thesis, Universität Konstanz, Konstanz, 2009.

10. Yanson, I. K.; Shklyarevskii, O. I.; van Ruitenbeek, J. M.; Speller, S. Aluminum Nanowires: Influence of Work Hardening on Conductance Histograms. Phys. Rev. B 2008, 77, 033411.

11. Pauly, F.; Viljas, J. K.; Huniar, U.; Häfner, M.; Wohlthat, S.; Bürkle, M.; Cuevas, J. C.; Schön, G. Cluster-based density-functional approach to quantum transport through molecular and atomic contacts. New J. Phys. 2008, 10, 125019.

12. Bürkle, M.; Viljas, J. K.; Hellmuth, T. J.; Scheer, E.; Weigend, F.; Schön, G.; Pauly, F. Influence of vibrations on electron transport through nanoscale contacts. Phys. Stat. Sol. B 2013, 250, 2468-2480.

13. Lü, J.-T.; Brandbyge, M.; Hedegård, P. Blowing the Fuse: Berry's Phase and Runaway Vibrations in Molecular Conductors. Nano Lett. 2010, 10, 1657-1663.

14. Lü, J.-T.; Hu, B.-Z.; Hedegård, P.; Brandbyge, M. Semi-classical generalized Langevin equation for equilibrium and nonequilibrium molecular dynamics simulation. Prog. Surf. Sci. 2019, 94, $21-40$.

15. Lü, J.-T.; Christensen, R. B.; Wang, J.-S.; Hedegård, P.; Brandbyge, M. Current-Induced Forces and Hot Spots in Biased Nanojunctions. Phys. Rev. Lett. 2015, 114, 096801.

16. Chen, R.; Matt, M.; Pauly, F.; Nielaba, P.; Cuevas, J. C.; Natelson, D. Shot noise variation within ensembles of gold atomic break junctions at room temperature. Journal of Physics : Condensed Matter 2014, 26, Article Number: 474204.

17. Cui, L.; Jeong, W.; Hur, S.; Matt, M.; Klckner, J. C.; Pauly, F.; Nielaba, P.; Cuevas, J. C.; Meyhofer, E.; Reddy, P. Quantized thermal transport in single-atom junctions. Science 2017, 355, 1192-1195.

18. Hellwege, K.-H., Olsen, J. L., Eds. Landolt-Börnstein - Group III Condensed Matter - Metals: Phonon States of Elements. Electron States and Fermi Surfaces of Alloys; Springer-Verlag, 1981. 